\title{
In vivo evaluation of ziram induced acute toxicity on pathomorphology of broiler chicken
}

\author{
Majid Shafi $^{1 *}$, Shayaib A. Kamil ${ }^{1}$, Masood S. Mir ${ }^{1}$, S. Adil ${ }^{2}$, Showkat A. Shah ${ }^{1}$ and Mir \\ Manzoor $^{1}$ \\ ${ }^{1}$ Division of Veterinary Pathology, Faculty of Veterinary Science \& Animal Husbandry, Sher-e-Kashmir \\ University of Agricultural Sciences \& Technology of Kashmir, Shuhama-190006 (J\&K), INDIA \\ ${ }^{2}$ Division of Livestock Production \& Management, Faculty of Veterinary Science \& Animal Husbandry, \\ Sher-e-Kashmir University of Agricultural Sciences \& Technology of Kashmir, Shuhama-190006 (J\&K), INDIA \\ "Corresponding author. E-mail: majidvetpatho@gmail.com
}

Received: March 10, 2016; Revised received: October 6, 2016; Accepted: November 25, 2016

\begin{abstract}
Fungicides are usually used in agriculture and often find their way in poultry feed. Therefore, a study was undertaken to study the in vivo effect of one such fungicide (ziram) induced intoxication on pathomorphology of broiler chicken. After 2 weeks of age the birds were given fungicide (Ziram) in feed as a single oral dose of $100 \mathrm{mg} / \mathrm{kg}$ body weight. Out of 10 birds, 3 died due to ziram intoxication (30\% mortality rate). Birds that died rapidly showed pronounced neurological signs like convulsions. The carcasses of ziram intoxicated birds appeared dehydrated and their mucous membrane was pale in colour. Marked vascular congestion was observed in brain on gross examination. The livers showed congestion and haemorrhages with necrotic foci. Kidneys and lungs had ecchymotic haemorrhages and heart revealed gelatinization of pericardium, distention and pericarditis. Atrophy of bursa of Fabricius and thymus; hypertrophy of thyroid was found. Histopathological examination revealed neuronal degeneration and necrosis associated with mild gliosis in brain. Lungs, pericardium and epicardium had severe congestion and there was degeneration with separation of myofibers. Glomeruli were congested and frequently revealed hypercellularity. There were sinusoidal congestion and varying degrees of hepatocellular degeneration. Bursa revealed mild depletion of lymphoid cells in few lobules while as thymus showed hypoplasia with depletion of lymphocytes. Thyroid had mild mononuclear cell infiltration and caecum showed marked necrosis and denudation of the mucosa. In conclusion, the depletion of lymphoid tissue from lymphoid organs was suggestive of immunosuppressive and immunomodulatory effects of ziram toxicity in broiler chicken.
\end{abstract}

Keywords: Broiler chicken, Immunosuppression, Lymphoid Depletion, Toxicity, Ziram

\section{INTRODUCTION}

Fungicides in agriculture are used to protect tubers, fruits and vegetables during storage and/or applied directly to ornamental plants, trees, field crops, cereals and turf grasses (Gupta \& Aggarwal, 2007). Dithiocarbamates (DTC) are type of fungicides which were first introduced for commercial applications during World War II (Ware and Whitacare, 2004). Besides their wide use as fungicides for treatment of crops, vegetables, seeds, and ornamental plants, they are also used as accelerators in the rubber industry and biocides in many household products (Edwards et al., 1991; Kamrin, 1997).

Ziram is analogous dialkyl DTC with differences in their groups used in preharvest agricultural applications. It is zinc dimethyldithiocarbamate, also known as methyl cymate (Osweiler et al., 1985). Ziram has been proven more toxic than ferbam and thiram in adult fowl (Rasul and Howell, 1974). These fungicides often find their way in poultry feed. Although there are benefits of using these pesticides but on the other hand these fungicides pose a potential threat to humans, birds and other animals. Fungicides toxicity with chlorpyrifos has been reported to significant decline the humoral immunity of broiler chicken (Kammon et $a l ., 2010 \mathrm{~b}$ ). As no literature regarding ziram toxicity in broiler chicken could be found, therefore, a study was undertaken with the objectives of studying the in vivo effect of induced ziram intoxication on pathomorphology of broiler chicken.

\section{MATERIALS AND METHODS}

Broiler chicken (10) in good health was procured from the market and used for the study. The experimental protocol was approved by Institutional Animal Ethics Committee vide no.AU/FVSc/C-09/2012/0476-88. All the chicks were vaccinated against prevalent diseases. The chicks were given Furasol at $1 / 2$ gram per litre of water for 5 days in the first week of experiment so as to prevent them from coccidiosis. The chicks were given broiler starter ration for two weeks followed by broiler finisher as per standards. After 2 weeks of age 
the birds received fungicide (Ziram) in feed. The route of toxication was selected as oral so as to simulate the natural condition in which birds are exposed for the toxicity under field conditions. The dose rate was selected on the basis of LD50.The LD50 of ziram in birds is $100 \mathrm{mg} / \mathrm{kg}$ body weight (Kamrin, 1997), so a single oral dose of ziram at $100 \mathrm{mg} / \mathrm{kg}$ body weight (LD50 dose) was given to the experimental birds.

The experimental birds were monitored during the entire period of experiment for clinical signs. All the sacrificed birds along with those died during the experiment were subjected to detailed postmortem examination. Gross lesions if any in different organs like thyroid, liver, spleen, heart, lungs, bursa of Fabricius, kidneys and intestine were recorded. For histopathological studies, pieces from above described internal organs were collected in $10 \%$ buffered formalin and the tissues were processed routinely and stained with Haematoxylin and Eosin as recommended by Lillie (1954).

\section{RESULTS AND DISCUSSION}

Clinical signs of ziram toxicity: The intoxicated birds were dull and depressed followed by anorexia. About half an hour after intoxication, the birds layed down and lacrimation was observed. The affected birds stood

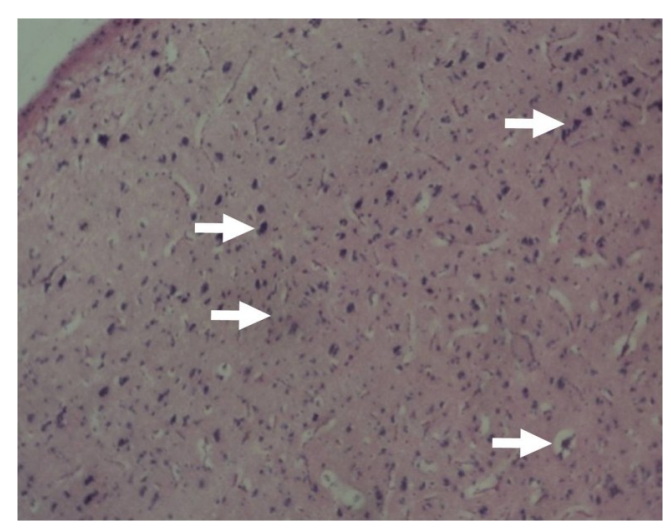

A

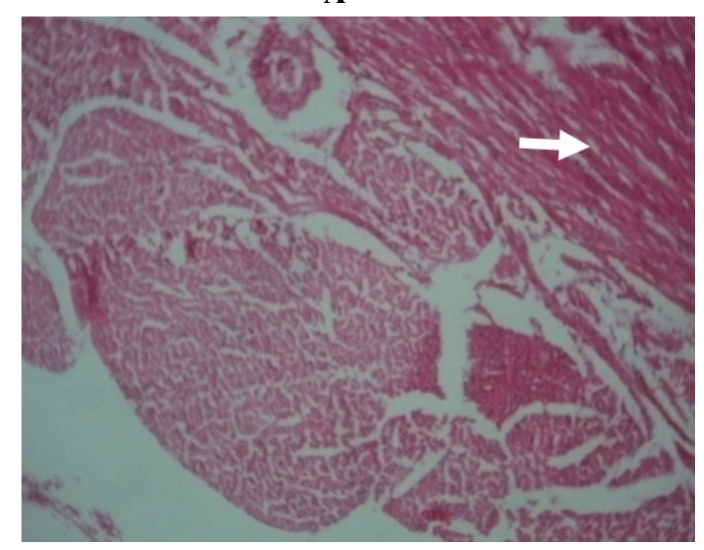

C motionless with head sunk onto the chest and both eyes closed. There was hyper salivation with decreased respiratory rate, pupil was dilated, and birds showed a staggering gait. Also, the intoxicated birds vomited suddenly and passed undigested food in faeces. Out of 10 birds, 3 died due to ziram intoxication (30\% mortality rate). The marked clinical signs observed in acute intoxicated birds included huddling, dullness, appeared listless, depressed and showed laboured breathing. The birds were reluctant to move and stood with ruffled feathers and cyanosed combs. Some of the affected birds also revealed lameness, enlarged hock joints and sternal recumbency. After 5-6 hours, birds exhibited increased thirst, droopy wings, and diarrhoea. Birds that died rapidly showed pronounced neurological signs like convulsions. The mucus membranes of the affected birds were pale and emaciated. The birds which died fell down suddenly and death occurred within one hour due to respiratory failure. Similar signs have been reported by Kaya and Bilgili (1998) with thiram toxicity in rabbits; Prasad and Koley (2006) in carbamate insecticide toxicity in ruminants; Hayes et al. (1991) in ziram intoxicated rats and Ema (1994) in carbamate intoxicated rats.

Gross pathology of ziram toxicity: The carcasses of ziram intoxicated birds appeared dehydrated and their

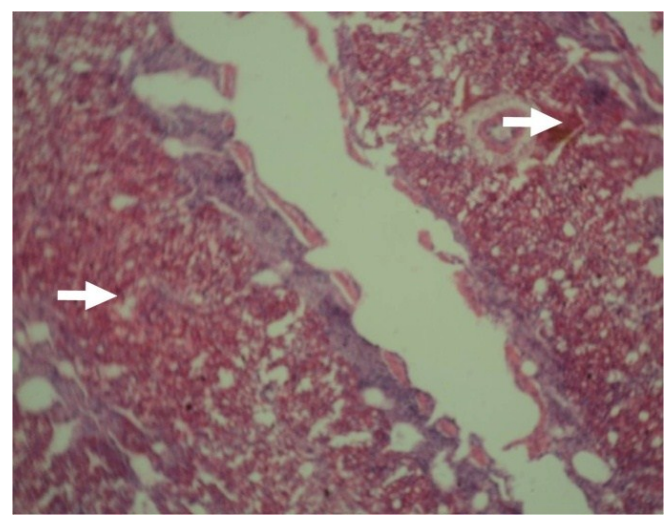

B

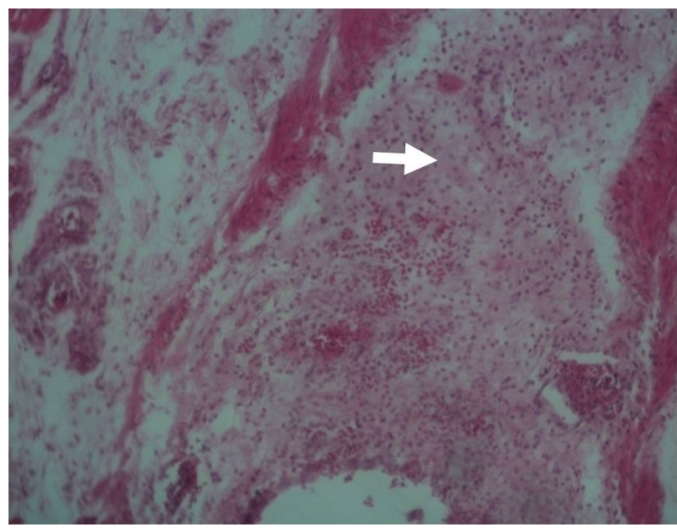

D

Contd..... 
Majid Shafi et al. / J. Appl. \& Nat. Sci. 8 (4): 2206-2211 (2016)

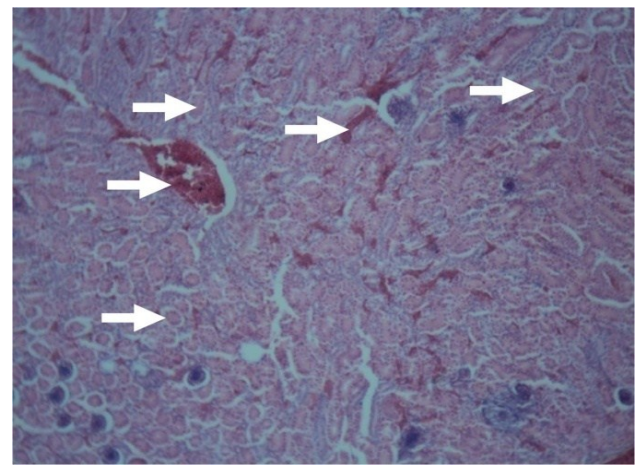

$\mathbf{E}$

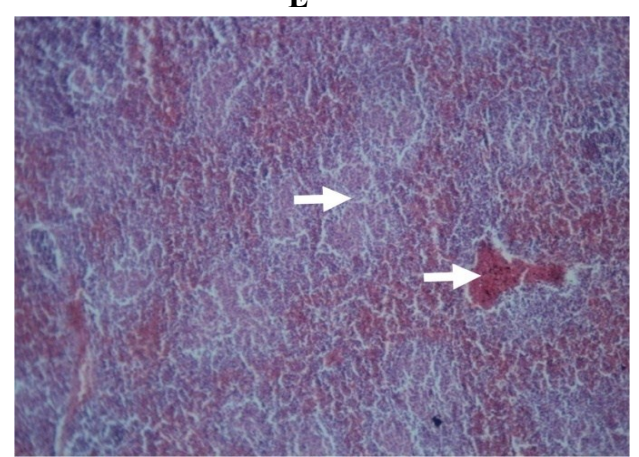

G

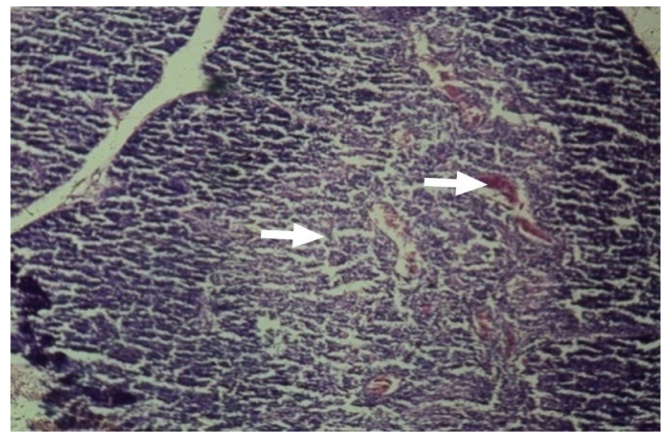

I

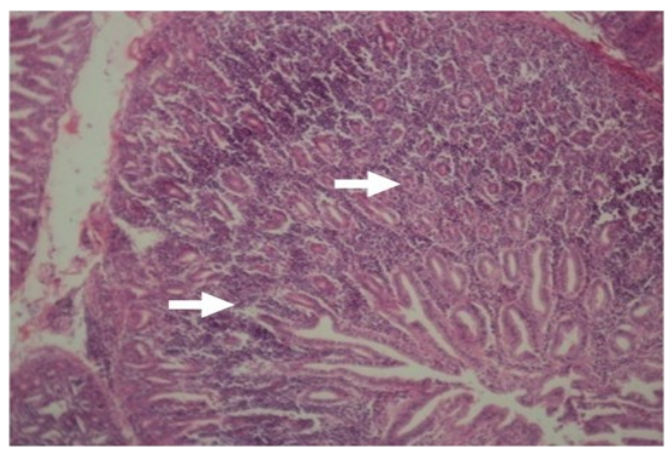

$\mathbf{K}$

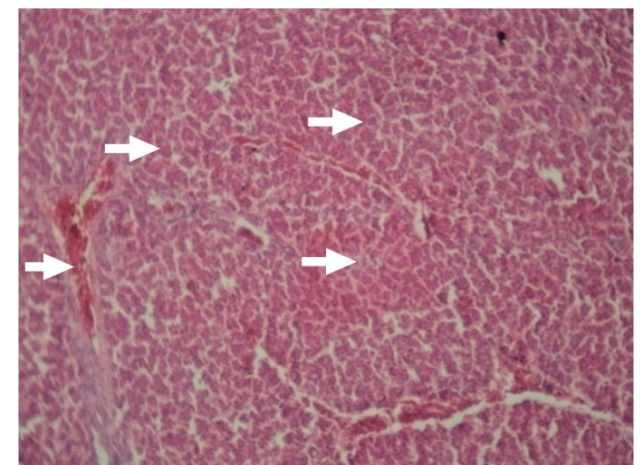

$\mathbf{F}$

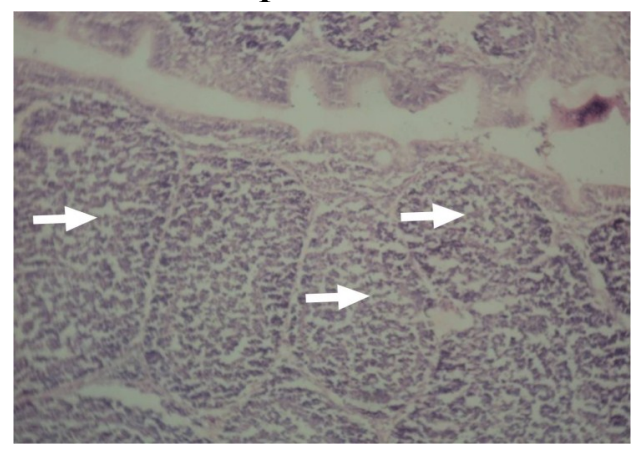

H
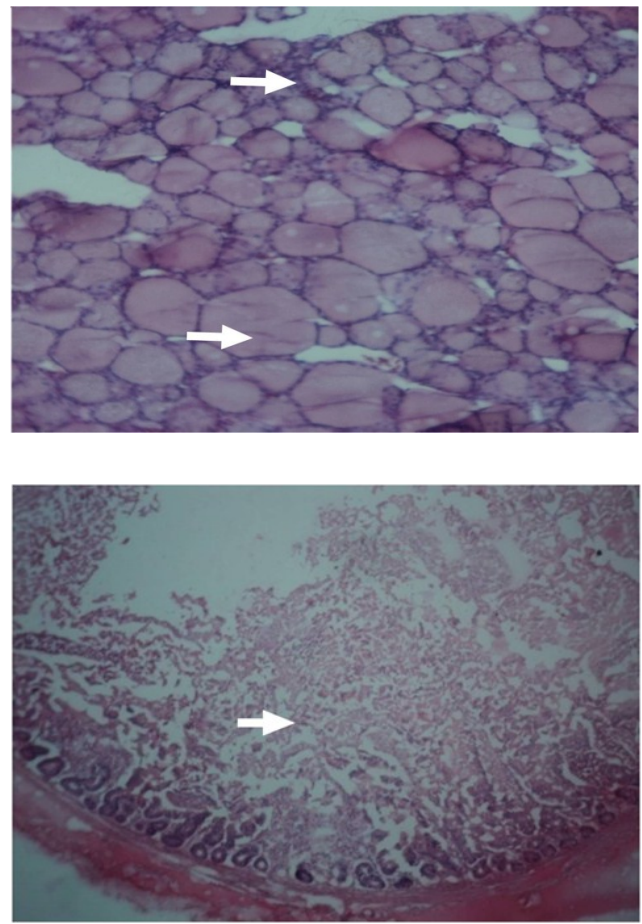

$\mathbf{L}$

Fig. 1. Histopathology of Ziram toxicity in broiler chicken. A. Cerebrum showing neuronal degeneration, necrosis, satellitosis and neuronophagia (H\&E 10X); B. Lung showing severe vascular congestion (H\&E 10X); C. Heart showing degenerative changes with separation of myofibers (H\&E 10X); D. Crop showing generalized edema with leukocytic infiltration (H\&E $10 X)$ E. Kidney showing vascular congestion and toxic nephrosis with loss of tubular epithelium (H\&E 10X) F. Liver showing vascular congestion and hepatocellular degeneration (H\&E 10X); G. Spleen showing vascular congestion, hemorrhages and loss of lymphoid cells (H\&E 10X); H. Bursa showing mild depletion of lymphoid cells (H\&E 10X);I. Thymus showing congestion, and mild lymphoid depletion (H\&E 10X).; J. Thyroid showing follicles distended with colloid material, and mild mononuclear cell infiltration (H\&E 10X); K. Proventriculus showing degeneration and necrosis of mucosa (H\&E 10X); L. Caecum showing marked necrosis and denudation of the mucosa $(H \& E 10 X)$. 
mucous membrane was pale in colour. The crop was distended, gelatinized, appeared transparent and was filled with mucinous material. There was loss of musculature with keel bone appearing sharp and distinct with gelatinized material. Marked vascular congestion was observed in brain on gross examination. The livers from intoxicated birds appeared larger in size and revealed congestion and haemorrhages varying from petechiae to suffusions. On palpation, the livers were generally firm and revealed creamy white necrotic foci on the surface. Cut surfaces revealed focal areas which were light coloured in few cases. Kidneys were generally rough, congested and revealed ecchymotic haemorrhages on the surface. Lungs appeared emphysematous. Congestion and ecchymotic haemorrhages were noted predominantly in the right lung while left lung in some cases revealed suffusions and areas of red hepatization. Heart revealed gelatinization of pericardium. In birds which died following intoxication, heart was generally distended and revealed pericarditis. Grossly, spleen revealed pinpoint necrotic spots with varying degrees of vascular congestion and haemorrhages in the birds of acute toxicity groups. Hemorrhages and atrophy was prominent in bursa of Fabricius in ziram imtoxicated birds which was easily visualised on gross examination. Thymus in ziram intoxicated birds appeared to be reduced in size. Slight increase in size of thyroid of ziram intoxicated birds was noted. Proventriculus appeared enlarged and oedematous with presence of serous exudates in the lumen.

\section{Histopathological examination}

Brain: There was a marked leptomeningial vascular congestion along with perivascular edema. Congestion was also noted in choroid plexus and cerebral microvasculature. Varying degrees of neuronal degeneration and necrosis associated with mild gliosis especially in the perivascular areas together with satellitosis and neuronophagia were also found (Fig. 1A). The necrotic small and large pyramidal cells appeared shrunken and stained darker with haematoxylin. More or less similar changes were reported by Hodge et al. (1952) in ziram toxicity of albino rats; Mahmoud (1997) and Marzouk et al. (2006) and in pesticide and herbicide toxicity of freshwater fishes.

Lungs: Microscopically, the lungs revealed severe generalized congestion and necrosis (Fig. 1B). In most of the cases air vesicles were filled with edematous fluid. The walls of the air vesicles were also moderately thickened. In addition, presence of severe emphysema due to rupture of air vesicles was evident in few birds. In other cases bronchi and bronchioles were filled with large amount of purulent exudate. Focal bronchopneumonia characterized by infiltration of lymphocytes predominantly in air vesicles, bronchi and bronchioles was evident in few cases. These lesions observed in the present study are in line with those observed by Gorizontoa et al. (1979) in mice and rats after the exposure to pyridinol carbamate; Maita et al. (1991) in rats and dogs and Novitsky et al. (2011) in rats with thiram toxicity.

Heart: The vascular congestion was observed in pericardium and epicardium. The heart generally showed degeneration with separation of myofibers (Fig. 1C). The vascular congestion in the epicardium, myocardium and endocardium of birds were also observed by Hart (1987). Maita et al. (1991) reported that thiram toxicity in rats resulted in Zenkers degeneration with separation of myofibers, thus confirming the results of present study. Most of the cases revealed thickening of epicardium and myocardium characterized by infiltration of mononuclear cells. Marked degeneration of myocardium was also evident in the auricular wall as was reported by Vanvleet and Ferrans (1994) in atrazine toxicity of rats.

Crop: Crop revealed generalized edema and marked degeneration of mucosa (Fig. 1D). Microscopical examination of crop tissues revealed presence of lymphoid aggregates in the walls. Histopathology in few cases further revealed severe desquamation of mucosa together with proliferation of stratified squamous epithelium. These lesions could be correlated with those observed by Najmus et al. (2010) in atrazine toxicity of broiler chicks.

Kidneys: Examination of kidneys revealed vascular congestion and degeneration with loss of tubular epithelium (Fig. 1E). Glomeruli were congested and frequently revealed hypercellularity and reduced periglomerular space. Renal tubules in the cortical and medullary region revealed degenerative changes characterized by swollen epithelium with indistinct cell boundaries and increased eosinophilia. Frequently necrosis and loss of tubular epithelium was noted. The pathological changes like cellular swelling and vacuolar changes in the lining epithelium of convoluted and collecting tubules were observed in acute thiram toxicity group of 60 weeks old white leghorn layers (Nageswara et al., 1996) and also by Sheftel (2000) in thiram intoxicated rabbits.

Liver: There were sinusoidal congestion and varying degrees of hepatocellular degeneration (Fig. 1F). Focal to diffuse areas of necrosis characterized by pyknosis and karyolysis of nucleus in hepatocytes associated with infiltration of lymphocyte and macrophages were observed in few cases. Similar changes have been reported in rats by Kurata (1981) and in ruminants by Sandhu and Barar (2009) associated with thiram carbamate toxicity.

Spleen: Spleen showed varying degree of vascular congestion, haemorrhages and necrosis. At places complete loss of lymphoid cells was evident (Fig. 1G). In some cases central arteries showed fibromuscular dysplasia characterized by massive proliferation of endothelial cells. These changes in the spleen are in accordance with those reported by Foster et al. (1979) and 
Jeong et al. (1998) in ethyl carbamate toxicity of mice.

Bursa: Histopathologically, bursa revealed mild degenerative changes in lymphoid cells along with the congestion. Mild depletion of lymphoid cells in few lobules was also evident in a few birds (Fig. 1H). These changes coincide with the findings of Khurana and Chauhan (2005) and Umesh et al. (2004) in pyrethroid toxicity of broiler chicks and pesticide toxicity of sheep respectively.

Thymus: Hypoplasia, decrease in size of the follicles with depletion of lymphocytes in the lymphoid follicles and congestion and haemorrhages in thymus were found (Fig. 1I). More or less similar changes have been reported by Songur et al. (2005) in carbendazim toxicity of rats and Cha et al. (2000) in ethyl carbamate toxicity of mice.

Thyroid: Generally thyroid revealed varying degree vascular congestion, haemorrhages and mild mononuclear cell infiltration (Fig. 1J). Histopathology of thyroid in few cases also revealed severe desquamation of epithelial cells and fibrous tissue proliferation. Similar findings were reported by Lakshman et al. (2002) in thiram toxicity of broiler chicken.

Proventriculus: Microscopically, the succumbed birds generally showed degeneration and desquamation of epithelial lining of the mucosal folds with presence of cellular debris in the lumen of proventriculus (Fig. $1 \mathrm{~K})$. Besides in a few cases necrotic lesion and hyperplasia of the parietal cells were also observed. Verma et al. (1999) also reported similar changes to some extend in mancozeb toxicity of broilers.

Intestine: The lining epithelium of intestinal mucosa generally revealed degeneration with the presence of mucoid exudate in the lumen. Frequently marked denudation of mucosa was also noted. Focal mononuclear cell infiltration was noted in submucosa in few cases. Caecum mostly revealed marked necrosis and denudation of the mucosa (Fig. 1L). In other birds hypertrophy of caecal wall, mild infiltration of mononuclear cells, focal degeneration and desquamation of mucosal epithelium was also evident. Similar results were observed by Gosselin et al. (1984) and Hayes et al. (1991) in ziram toxicity of rats.

\section{Conclusion}

It could thus be concluded that acute poisoning of birds at $100 \mathrm{mg} / \mathrm{kg} \mathrm{b.wt}$. of ziram has a toxic effect on liver, kidneys, thyroid and lymphatic organs. The depletion of lymphoid tissue from lymphoid organs was suggestive of immunosuppressive and immunomodulatory effects of ziram toxicity. Birds could get accidentally exposed to ziram and may prove harmful to human population as well following consumption of such birds. Thus, the safe and effective application and disposal of fungicides which may include proper dosage and taking due care of drug withdrawal period must be strictly ensured.

\section{REFERENCES}

Cha, W. and Jeong, C. (2000). Immunotoxicity of ethyl carbamate in female mice: Toxicol. Lett., 115(3): 173-181

Edwards, I.R., Ferry, D. and Temple, A. (1991). Fungicides and related compounds toxicity. Pesti. Toxi., pp: 4-12

Ema (1994). Developmental toxicity evaluation of zinc dimethyldithiocarbamate (Ziram) in rats. Bull. Enviro. Conta. Toxi., 53(6): 930-936

Foster, T. and Akhtar, M. (1979). Metabolism of atrazine by solubile fraction from chicken liver homogenates. $J$. Agric. Food Chem., 27: 300-302

Gorizontova, P. and Shvarts, G. (1979). Effect of pyridinol carbamate on microvascular permeability in the lungs, skin, and mesentery. Bull. of Exper. Bio., 88 (3): $971-974$

Gosselin, R.E., Smith, R.P. and Hodge, H.C. (1984). Clinical Toxicology of Commercial Products. 5th ed. Balti, pp. 314.

Gupta, P.K. \& Aggarwal, M. (2007). Toxicity of fungicides. In: Veterinary Toxicology, Gupta, R.C. (Ed.), 1st ed., 587-601, Elsevier, 978-0-12-370467-2, New York.

Hart, L. (1987). The toxicity of atrazine in fowls. Aust. Vet. J., 17: 69-71

Hayes, W.J. and Laws, J. (1991). Effects of oral administration of ziram in rats. Handbook of Pesticide Toxicology. Classes of Pesticides, pp. 1446.

Hodge, H., Maynard, A. and Jones, K. (1952). Acute and short-term oral toxicity tests of ziram. J. Am. Pharm. Assoc., 41(12): 662-665

Jeong, T. and Roh, J. (1998). Effects of ethyl carbamate and its metabolites on the antibody response in splenocyte cultures from female Balb/C mice. Immumopharmacol. Immunotoxicol., 18 (1): 91-103

Kammon, A.M., Brar, R.S., Sodhi. S., Banga, H.S., Nagra, N.S. and Singh, J. (2010b). Ameliorating effect of vitamin $\mathrm{C}$ on immunological implications induced by chronic chlorpyrifos toxicity in broilers. Libyan Vet. Med. J., 1 (1): 164-180

Kamrin, M.A. (1997). Pesticide profile, Toxicity, environmental impact and fate. CRC Press, Boca Raton.

Kaya, S. and Bilgili, A. (1988). Effect of thiram in rabbit. Vet. Toxicol., 975

Khurana, R. and Chauhan, S. (2005). Immunopathological effects of pesticides on lymphoid organs in sheep. $J$. Immunopatho., 7 (1): 64-68

Kurata, Y. (1981). Subchronic toxicity of thiram was investigated in rats. Bulletin Nation. Inst. Hyg. Sci., 4 (98): 6976

Lakshman, M., Ahemed, S.R. and Sarma, B. (2002). Effect of dietary tetra methylthiuramdisulphide (TMTD) on the development of experimental tibial dyschondroplasia (TD) in chicks. Ind Vet. Patho., 26 (6): 724-728

Lillie, R.D. (1954). Histopathologic Technic and Practical Histochemistry. New York: Blakiston Co.

Mahmoud, O.H. (1997). Effect of pollution with pesticides on blood constituents of freshwater fishes. M. V. Sc. Cairo University, Facuty of Veterinary Medicine

Maita, K. and Shirasu, Y. (1991). Chronic toxicity studies with thiram in Wistar rats and beagle dogs. Fundam Appl Toxicol. Toxicology Division, Inst. Envir. Toxi., 4: 667-686

Marzouk, M.S., Hafez, M.A. and Enas, R. (2006). Effect of a 
currently used herbicide on the health status of Oreochromis niloticus in Egypt. Egypt J. Agric. Res., 84 (1): 417-433

Nageswara, A. and Srilatha, C. (1996). Effects of thiram on performance of layers. Indian J. Poult. Sci., 31 (3): $173-180$

Najmus, (2010). Haematological effects of chronic toxicity of atrazine fungicide in broilers. IOSR J. Agri. Vet. Sci. 4: $19-22$

Novitskiy, V. and Eshchenko, A. (2011). Effects of chelating agents dithizone and sodium diethyldithiocarbamate on zinc content in Paneth cells and prostate gland cells on rats. Bull. Exp. Biol. Med., 152 (2): 180-183

Osweiler, G.D., Carlson, T.L., Buck, W.B. and Von Gelder, G.A. Eds. (1985). Organic synthetic fungicides. In Clinical and Diagnostic Veterinary Toxicology, 3rd ed., 231-242, Kendal/Hunt, 0-8403-3332-3, Dubuque

Prasad, V.V. and Koley, K.M. (2006). Carbamate toxicity in ruminants. Vet. Toxi., pp.1304-1305
Rasul, A.R. and Howell, J.M. (1974). The toxicity of some diiithiocarbamate compounds in young and adult domestic fowl. Toxicol. Appl. Pharma, 31(1): 63-78

Sandhu, H.S. and Barar, R.S. (2009). Carbamate toxicity in ruminant Textbook of Veterinary Toxicology, 2: 195-197

Sheftel, V.O. (2000). Thiram study on rabbits. Mig. Toxi., 22: 548

Songur, S. and Barlas, N. (2005). Dose-dependent effects of carbendazim on rat. Int. Bio. Funct., 23(6): 457-460

Umesh, K. and Jadhao, B. (2004). Pathophysiological Effects of Chronic Toxicity with Synthetic Pyrethroid, Organophosphate. Toxicol Pathol., 32: 364

Vanvleet, J.F. and Ferran, J. (1994). Myocardial diseases of animals. Am. J. Pathol., 124: 98-178

Verma, D. and Taluja, J.S. (1999). Effect of mancozeb in fowl Gallus domesticus. Ind. J. Ani. Sci., 69(11): 898-901

Ware, G.W. and Whitcare, D.M. (2004). The pesticide book. $6^{\text {th }}$ ed. Meister Pro Information Resources, Willoughby, $\mathrm{OH}$ 